\section{Functional cue learning under different probabilistic feedback conditions*}

\author{
L. F. W. de KLERK and S. OPPE
}

University of Leiden, Leiden, The Netherlands

This study was designed to investigate single-cue learning. The cue, the response (S's estimation of the criterion), and the reinforcement (criterion) were varied in discrete steps. Two different cue criterion relationships (linear and quadratic) and two levels of probabilistic feedback were used. The results indicated that (1) the linear function is more accessible than the quadratic function; (2) the task becomes more difficult when the criterion variance is increased. When the task requires the $S$ to learn the quadratic function, he first attempts to improve the linear orthogonal component of this rule and then tries to improve the quadratic component. This effect is explained in terms of the progression hypothesis, viz, that the Ss initially make use of the lower order components of the input and progress with practice to the higher order components.

There has been considerable investigation into the learning of information processing. The focus here will be on what Carroll (1963) has called functional learning. In a typical functional learning task, there usually is a scaled cue variable, $x_{i}(i=1, \cdots$ $n)$, a scaled criterion variable, $y_{i}(i=1$, $\cdots, n$ ), and a real valued function which determines the assignment of $y_{i}$ to $x_{i}$. The criterion for learning is the prediction of $y_{i}$, say $\tilde{y}_{1}$, when confronted with $x_{i}$.

According to the theory of functional learning, proposed by Brehmer (1971), the Ss must learn two things: They first have to discover the function relating criterion to cue values, and then they have to learn to use the function. As to the former stage of the learning process, $S$ is assumed to sample hypotheses about the structure of the task and to test them against the outcome (provided that feedback is given during training) until he has discovered the correct hypothesis.

There is a fair amount of evidence that the set of the total possible hypotheses is hierarchically structured. Studies in functional learning have shown (1) that linear functions are more accessible than nonlinear cue criterion relations (Brehmer, 1971; Carroll, 1963; De Klerk, De Leeuw, \& Oppe, 1966; Hammond \& Summers, 1965; Summers \& Hammond, 1966), and (2) that it is easier for $\mathrm{S}$ to learn positive linear functions than negative linear functions (Bjorkman, 1965; Brehmer, 1971; De Klerk et al, 1966; Eisler \& Spolander, 1970; Naylor \& Clark, 1968)

There is also some evidence that Ss start constructing "simple hypotheses" and that they progress-if

* This study was supported by a grant from the Netherlands Organization for the Advancement of Pure Research (Z.W.O.). necessary-towards more difficult and more complex hypotheses. Studies by Hammond \& Summers (1966) and by Summers \& Hammond (1965) have shown that in multiple cue learning tasks (involving both linear and nonlinear relations), linear processing precedes nonlinear processing. Naylor $\&$ Carroll (1966) have obtained evidence for the progression hypothesis in a perceptual-cognitive inference task, where the stimuli were parabolas drawn on Cartesian coordinates. The correct response for each parabola was based on an equation which involved the first and second derivates of each stimulus figure.

The present study is an attempt to test the validity of the progression hypothesis in a single-cue learning task, where both cue and criterion are varied in discrete steps. The specific purpose of this study is to investigate whether progression can be demonstrated by means of an orthogonal component analysis. The hypotheses are: When a nonlinear cue criterion relation is to be learned, there will be a progression from the lower (linear) orthogonal component to higher order (nonlinear) orthogonal components. This effeet is supposed to be dependent on the criterion variance.

\section{DESIGN}

Four experimental conditions were created by using two different functions and two levels of probabilistic feedback. The functions were $\left(A_{1}\right): y=x+2$, and $\left(A_{2}\right): 1 / 2 x^{2}=$ $2 \frac{1}{2} x+5$, respectively. For each cue value $x$ there was a probability distribution $p_{\mathrm{x}}(y)=p(Y=y \quad X=x)$ over the set of criterion values. $p_{Y}(y)$ must be interpreted as the probability that $\mathrm{y}$ is reinforced given $\mathrm{x}$.

In this study, the feedback distribution $\mathrm{p}_{\mathrm{x}}(\mathrm{y})$ was defined as:

$$
\begin{aligned}
p_{X}(y) & =p, Y=[f(x)+1 / 2 N-k]: X=x ! \\
& =\left\{\begin{array}{l}
C_{k}^{N} p^{k}(1-p)^{N-k}, \text { with } k=0 . \cdots, N \\
0 \text { otherwise }
\end{array}\right.
\end{aligned}
$$

where $\mathrm{p}=.5$

In one condition $\left(B_{1}\right), N=2$; in a second $\left(B_{2}\right), N=4$

It can be seen that the expected values of the feedback distributions are:

$$
e_{x}(y)=\sum_{k} C_{k}^{N} p^{N}[f(x)+1 / 2 N-k]=f(x)
$$

This means that the expected values varied with $x$ according to the above-mentioned first- and second-degree polynomials, respectively.

\section{MATERIALS}

For each experimental condition, the values of both $x$ and $y$ were represented along two perpendicular axes. Typically, values of $x$ (cue) were represented along the horizontal axis, whereas values of $y$ (criterion) were located along the vertical axis. The $y$-axis was divided into units of $1 \mathrm{~cm}$ and labeled from 0 (i.e:, the origin) to 10. No scale was present on the $x$-axis. The values of $\mathrm{x}$ and $\mathrm{y}$ were indicated by small arrows. The position of the arrow on the $x$-axis was varied from 0 to $12 \mathrm{~cm}$ from the origin in units of $2 \mathrm{~cm}$. Thus, there were seven different cue values.

The materials were presented to the $S$ via training booklets. For every experimental condition, five training booklets (labeled from I through V) were constructed. Every page contained the two orthogonal axes and two arrows which marked off the values of $\mathrm{x}$ and $\mathrm{y}$, respectively. Within each condition, the seven different $\mathrm{x}$-values were used 16 times. The corresponding values of $y$ were obtained from the feedback distribution, $p_{x}(y)$, that was constructed for the condition in question. Thus, the total number of $x-y$ pairs within every condition was 7 $x 16=112$. Booklet I contained 24 pairs; the other four booklets contained 22 pairs each. The pairs occurred in different random orders in every booklet.

The test materials were also presented via a booklet. One test booklet was used for all conditions. This booklet consisted of 112 pages. Every page contained the two orthogonal axes and only the value of $\mathrm{x}$. The 7 by $16 \mathrm{x}$-values occurred in a random order.

\section{PROCEDURE}

Every $S$ received five training booklets and one test booklet. They were instructed to take Booklet I first and to work through the pages in 
Table 1

The $x^{2}$ Measures Per Run Per Condition

\begin{tabular}{|c|c|c|c|c|c|c|}
\hline \multirow[b]{2}{*}{$r$} & \multicolumn{5}{|c|}{ Run } & \multirow[b]{2}{*}{ df } \\
\hline & 1 & 2 & 3 & 4 & 5 & \\
\hline \multicolumn{7}{|c|}{ Condition $A_{1} B_{1}$} \\
\hline 5 & 2.74 & 5.06 & 2.33 & 1.06 & 1.46 & 4 \\
\hline 4 & 3.28 & 6.29 & 2.70 & 3.76 & 2.06 & 8 \\
\hline 3 & 12.46 & 18.67 & 13.61 & 7.21 & 3.65 & 12 \\
\hline 2 & 15.57 & 18.99 & 17.85 & 7.75 & 6.63 & 16 \\
\hline 1 & 26.99 & 20.51 & 18.05 & 8.45 & 9.18 & 20 \\
\hline 0 & 395.59 & 397.93 & 381.70 & 380.99 & 409.90 & 24 \\
\hline \multicolumn{7}{|c|}{ Condition $A_{1} B_{=}$} \\
\hline 5 & .55 & 6.17 & .62 & .17 & 3.68 & 4 \\
\hline 4 & 2.42 & 6.89 & 3.22 & .74 & 4.92 & 8 \\
\hline 3 & 8.63 & 24.65 & 6.75 & 1.03 & 9.89 & 12 \\
\hline 2 & 9.61 & 25.74 & 7.07 & 1.94 & 12.52 & 16 \\
\hline 1 & 26.67 & 37.04 & 13.43 & 9.46 & 12.75 & 20 \\
\hline 0 & 329.97 & 421.65 & 470.33 & 384.57 & 413.94 & 24 \\
\hline \multicolumn{7}{|c|}{ Condition $A_{2} B_{1}$} \\
\hline 5 & 3.01 & 1.46 & 5.46 & 4.76 & 4.16 & 4 \\
\hline 4 & 10.05 & 3.43 & 7.63 & 6.94 & 5.88 & 8 \\
\hline 3 & 14.46 & 6.93 & 7.89 & 12.26 & 16.69 & 12 \\
\hline 2 & 21.38 & 23.88 & 12.81 & 16.99 & 26.37 & 16 \\
\hline 1 & 228.03 & 294.30 & 280.13 & 275.63 & 352.30 & 20 \\
\hline 0 & 370.29 & 388.43 & 361.34 & 360.23 & 420.53 & 24 \\
\hline \multicolumn{7}{|c|}{ Condition $A_{2} B_{2}$} \\
\hline 5 & .17 & .19 & 1.62 & .71 & 2.43 & 4 \\
\hline 4 & 1.79 & 5.84 & 3.18 & 2.53 & 4.47 & 8 \\
\hline 3 & 28.58 & 12.20 & 11.85 & 24.46 & 16.00 & 12 \\
\hline 2 & 72.86 & 21.94 & 22.33 & 37.49 & 20.54 & 16 \\
\hline 1 & 87.64 & 100.39 & 134.63 & 192.02 & 157.65 & 20 \\
\hline 0 & 285.60 & 280.65 & 248.30 & 259.87 & 276.95 & 24 \\
\hline
\end{tabular}

Table 2

The $x^{2}$ Values Added Over Runs

\begin{tabular}{|c|c|c|c|c|c|}
\hline \multirow[b]{2}{*}{ Degree } & \multicolumn{4}{|c|}{ Condition } & \multirow[b]{2}{*}{ df } \\
\hline & $A_{1} B_{1}$ & $A_{1} B:$ & $A_{2} B_{1}$ & $A: B=$ & \\
\hline 5 & 12.65 & 11.19 & 18.85 & 5.12 & 20 \\
\hline 4 & 18.09 & 18.19 & 33.93 & 17.81 & 40 \\
\hline 3 & 55.60 & 50.95 & 58.23 & $92.09 *$ & 60 \\
\hline 2 & 66.79 & 56.88 & 101.43 & $175.16^{*}$ & 80 \\
\hline 1 & 83.18 & 99.35 & $1430.39^{*}$ & $672.33^{*}$ & 100 \\
\hline 0 & $1966.11^{*}$ & $1984.46^{*}$ & $1900.82^{*}$ & $1351.37^{*}$ & 120 \\
\hline
\end{tabular}

Table 3

The $x^{2}$ Values Per Run Per Condition for the Orthogonal Polynomials of Degrees 1 and 2, Respectively*

\begin{tabular}{|c|c|c|c|c|c|c|}
\hline \multirow{2}{*}{$\begin{array}{l}\text { Con- } \\
\text { dition }\end{array}$} & \multirow[b]{2}{*}{ Degree } & \multicolumn{5}{|c|}{ Run } \\
\hline & & 1 & 2 & 3 & 4 & 5 \\
\hline \multirow{2}{*}{$A_{1} B_{1}$} & 1 & 50.72 & 33.79 & 24.67 & 34.45 & 16.46 \\
\hline & 2 & 17.03 & 1.43 & 1.80 & 1.90 & 3.00 \\
\hline \multirow{2}{*}{$A_{1} B_{2}$} & 1 & 93.64 & 38.26 & 7.86 & 64.89 & 30.17 \\
\hline & 2 & 14.88 & 10.47 & 5.15 & 7.51 & .08 \\
\hline \multirow{2}{*}{$A_{2} B_{1}$} & 1 & 16.08 & 10.16 & 7.28 & 11.06 & 17.05 \\
\hline & 2 & 73.50 & 21.94 & 21.04 & 36.12 & 17.42 \\
\hline \multirow{2}{*}{$A_{2} B=$} & 1 & 104.67 & 40.12 & 2.89 & 14.31 & 8.27 \\
\hline & 2 & 394.31 & 381.26 & 182.41 & 123.08 & 179.12 \\
\hline
\end{tabular}

*Each $x^{2}$ is connected with 4 df.

order. They were allowed to work at their own pace. During the test which immediately followed the learning phase, the Ss were asked to predict the value of $y_{i}$ when confronted with $x_{i}$ (i $=1, \cdots, 112$ ). The verbal numerical responses (being the integers from 0 to $10)$ were recorded by the E. After this during testing.

\section{SLBJECTS}

Sixteen Ss participated in this study. They were divided randomly into four groups with four $S$ s in each. They were unfamiliar with this type of experiment.

RESULTS
The numerical responses for each $\mathrm{S}$ for each run were summarized in an $n$ by $m$ frequency matrix, where $n$ is the number of different stimuli that could occur (7) and $m$ the number of different responses that could be given (11). De Leeuw (see appendix) has proposed to apply an analytic technique to these data which is based on a decomposition into orthogonal polynomials. This technique enables determination (by means of chi-square measures) of which polynomials significantly contribute to the mean responses. This analysis has been carried out for each $S$ per run. The results (independent chi-square measures) were added over the four Ss for each condition. These chi-square measures are given in Table 1 , and the effects of adding over runs are given in Table 2.

By using the same type of analysis, it was also possible to compare the polynomial coefficients with those based upon the optimal responses [i.e., the expected values $\left.e_{x}(y)\right]$. This analysis was carried out for the first three orthogonal polynomials. The results are given in Table 3 .

Finally, for ecch condition, the mean response per stimulus was computed. The results are presented graphically in Fig. 1.

It may be concluded that performance is better when Ss have to learn a linear rule than when they have to learn a quadratic rule. The ratio of the chi-square values that measure the discrepancy between actual and optimal performance for the quadratic and the linear conditions, respectively, is $\mathbf{F}=4.23$. This is significant at the $1 \%$ level. There is also a significant feedback effect. It turns out that the task is more difficult when the variance of the feedback distribution is increased. This effect is significant for the linear $(F=1.87 * *)$ as well as for the nonlinear conditions $\left(F=5.95^{* *}\right)$. DISCUSSION

The graphs of Fig. 1, showing the average performance in each of the four experimental conditions, clearly demonstrate that Ss did learn to use the correct type of function. From the data of Table 2, it can be concluded that under both linear conditions, the only significant value of chi square was for the zero component, indicating that Ss had learned to use a linear rule. When the task required the learning of a quadratic rule, performance was highly dependent upon the feedback 

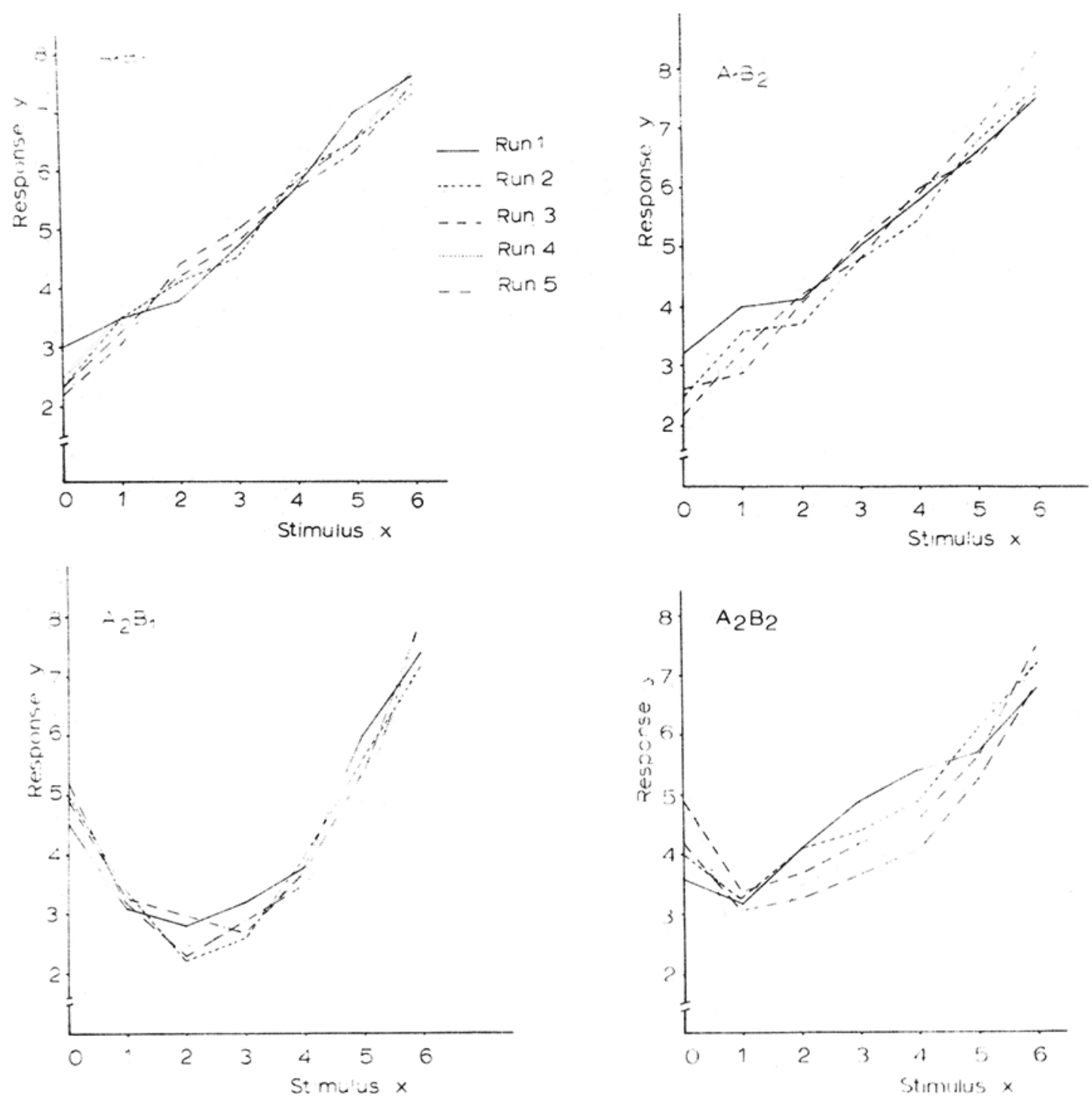

Fig. 1. The mean response per stimulus for each condition.

conditions used. The results for Condition $A_{2} B_{1}$ show that $S s$ did, indeed, employ a quadratic rule. Under Condition $A_{2} B_{2}$, the first four orthogonal polynomials did account for a significant part of the variance in the mean prediction responses. However, as may be seen in Table 1 , this effect is mainly due to poor performance during the early stages of learning. The results of the last run of this particular condition show that Ss eventually used a quadratic rule. From the data of Table 3 , it can be seen that the overall performance was significantly better when $S$ s had to learn a linear rule. Thus, when tested under the nonlinear conditions, performance deviated more from the optimum than when the linear function was used.
As to the nonlinear conditions $\left(A_{2} B_{1}, A_{2} B_{2}\right)$, it can be said that both the first- and the second-degree orthogonal polynomial decreased as a function of training. This effect is largely dependent upon the probability structure of the learning task. It turns out that Ss could not use outcome feedback to determine the correct rule as effectively and adequately when the criterion variance was large $\left(A_{2} B_{2}\right)$ as when it was small $\left(\mathrm{A}_{2}, \mathrm{~B}_{1}\right)$.

It can be concluded that in these nonlinear conditions, the $S$ s respond essentially according to a quadratic rule, even when they are tested for the first time (i.e., immediately after the first block of 24 learning trials). However, the results suggest that they first attempt to improve the linear orthogonal component of this rule and then try to improve the quadratic component. This finding gives support to the progression hypothesis, reported above, viz, that Ss initially make use of the lower order components of the input and progress with practice to the higher order components.

Finally, by inspection of the data for Conditions $A_{2} B_{1}$ and $A_{2} B_{2}$, it is apparent that increased variability of the feedback distribution makes it more difficult for the $S$ to learn the quadratic orthogonal component of the function than the linear component. This result gives further support for the progression hypothesis, which would predict that the increase of difficulty of the learning task affects the higher order 
components of the task to a larger extent than it does the lower order components.

\section{APPENDIX \\ Notation}

The values of the independent variable are denoted by $x_{i}(i=1, \cdots, n)$, and the values of the dependent variable (i.e., the numerical responses) by $y_{j}(j=1, \cdots, m)$.

$P$ stands for the matrix of response probabilities $\left(\mathrm{p}_{i j}: \mathrm{i}=1, \cdots, \mathrm{n} ; \mathrm{j}=1, \cdots, \mathrm{m}\right)$.

$\mathrm{Z}$ represents the matrix of orthogonal polynomials on $\times\left(z_{i t}: i=1, \cdots, n: t=0\right.$ $\cdots, n-1$ )

\section{Hypotheses}

H(a): $\mathbf{Z} \cdot \mathbf{P y}=\alpha$ with $\alpha$ completely or partially specified. In particular:

$$
H_{p}: Z^{\prime} P_{y}=\left\lceil\frac{\alpha}{0}\right\rfloor
$$

where the first $(p+1)$ elements $a$ are unrestricted.

Define: $\hat{\alpha}=z^{\prime} \hat{\mathrm{p}} \mathrm{y}$

$$
\text { Test }
$$

Assmptotically $\alpha$ is distributed as $\eta(\alpha, \phi)$ with $\varphi=Z^{\prime} W Z$, where $W$ is a diagonal matrix with:

$$
w_{i i}=\frac{1}{n_{i}} y^{\prime} V_{i} y=\frac{1}{n_{i}}\left[\Sigma y_{j}^{2} p_{i j}-\left(\Sigma y_{j} p_{i j}\right)^{2}\right]
$$

Define: $;=\mathrm{z}$ itz with

$$
\left.\hat{\mathrm{w}}_{i \mathrm{i}}=\frac{1}{n_{i}} \mid \Sigma y_{j}^{2} p_{i j}-\left(\sum_{j} p_{i j}\right)^{2}\right\} .
$$

Then the Nerman Wald test of $H(\alpha)$ is to compare $\delta=(\hat{\vec{\alpha}}-Q)^{\prime} \hat{\phi}^{+}(\hat{\vec{u}}-\alpha)$ with a chi square with $r=$ rank $(O)$ degrees of freedom (cf. Wald, 1943: Nerman, 1949: Bhapkar, 1961, 1966). By $\hat{\phi}^{+}$is meant a (one condition) generalized inverse of $\$$

\section{REFERENCES}

BHAPKAR, V. P. Some tests for categorial data. Annals of Mathematical Statistics. $1961,32,72-83$.

BHAPKAR, V. P. A note of the equivalence of two test-criteria for hypotheses in categorical data. Journal of the American Statistical Association. 1966. 61. 228-237.

BJORKMAN, M. Learning of linear functions: Comparison between a positive and a negative slope. Reports from the Psychological Laboratories. University of Stockholm. No. 183, 1965.

BREHMER, B. Subject's ability to use functional rules. Psychological Report No, 43. University of Umea, Sweden. 1971 .

CARROLL, J. D. Functional learning: The learning of continuous functional mappings relating stimulus and response continua. Rusedreh Bulletin No R B-tia-2ti. Educational Testing Service, Princeton. New Jersey. 1963.

DE KLERK. L. F. II., DE LEELW.J. \& OPPE. S. Funktionet leren. Hypothest. $1966,11,10-19$

FISLER, $H$ \& SPOLANDER, $k$, On the sign of slope in the learning of lineat fuuctions. Scandinatian Journal of Psycholugs. 1970,11, 176-184.

HAMMOND, K. R., \& SUMMERS. D. A. Cognitive dependence on linear and non-linear cues. Psvchological Reriew. 1965, 72, 215-224.

NAYLOR, L. C.. \& CARROLL, R. M. A test of the progression-regression hypothesis in a cognitive inference task. Organizational Beharior \& Human Performance. 1969.4. 337-352.

NAYI,OR,J.C.. \& CLARK. R. D. Intuitive inference strategies in interval learning tasks as a function of validity. magnitude and sign. Organizational Behavior \& Human Performance, 1968, 3. 378-399.

NEYMAN.J. Contribstions to the theory of the $x^{2}$ test. Proceedings of the First Berkeley Svmposium, 1949, 239-273.

SUMAERS, D. A., \& HAMMOND. K. R. Inference behavior in multiple-cue tasks involving both linear and non-linear relations. Journal of Experimental Psychology, 1966,71,751-757.

WALD.A. Tests of statistical hypotheses concerning several parameters when the number of observations is large. Transactions of the American Nathematical Society, 1943, 426-482. 